\title{
Research on Saponin Active Ingredient of Stir-Fried Dolichos Lablab L. Kernel for Treatment of Type-2 Diabetes Based on UHPLC-Q-Exactive Orbitrap MS and Network Pharmacology
}

Han Jun ( $\boldsymbol{0} 729229056 @ q q . c o m)$

Beijing Tcmages Pharmaceutical Co Ltd https://orcid.org/0000-0002-3634-3991

Liangzi Fang

Hunan Academy of Chinese Medicine: Hunan University of Chinese Medicine

Qinfang Zheng

Hunan University of Medicine

\section{Research}

Keywords: UHPLC-Q-Exactive Orbitrap MS, network pharmacology, stir-fried Dolichos lablab L. kernel, type-2 diabetes, molecular docking, molecular dynamics simulation, ligand channels

Posted Date: November 3rd, 2020

DOI: https://doi.org/10.21203/rs.3.rs-98583/v1

License: (c) (1) This work is licensed under a Creative Commons Attribution 4.0 International License.

Read Full License 


\title{
Research on Saponin Active Ingredient of Stir-fried Dolichos lablab L. Kernel for Treatment of Type-2 Diabetes based on UHPLC-Q-Exactive Orbitrap MS and Network Pharmacology Jun Han, Liangzi Fang, Qinfang Zheng
}

\begin{abstract}
Background: Although the clinical effect of stir-fried Dolichos lablab L. kernel has been approved in modern traditional Chinese medicine, existing associated studies mainly focus on its clinical studies and chemical ingredients. However, there are few studies on pharmacodynamics material basis and molecular mechanism of stirfried Dolichos lablab L. kernel in treatment of type-2 diabetes(T2DM), thus restricting the further development and utilization of stir-fried Dolichos lablab L. kernel.Methods: A qualitative analysis on saponin chemical ingredients of stir-fried Dolichos lablab L. kernel was performed using UHPLC-Q-Exactive Orbitrap MS. A total of 10 saponin ingredients were selected. Moreover, target screening, biological process and metabolism pathway analysis were accomplished by network pharmacology. Four key proteins (EGFR, IGF1, MAPK1 and PIK3R1) of type-2 diabetes were selected for molecular docking verification with saponin ingredients. Specifically, molecular dynamics simulation of ingredients which have strong bindings with proteins was conducted. Results: In this study, 16 saponin ingredients were identified from stir-fried Dolichos lablab L. kernel. There were 91 intersection targets and the KEGG pathway enrichment involved 20 relevant pathways. According to the molecular docking verification, saponin ingredients of stir-fried Dolichos lablab L. kernel can form stable binding with key protein targets. The molecular dynamics simulation further verifies stability and reasonability of the docking results. Conclusions: This study provides references to identification of efficient ingredients of stir-fried Dolichos lablab L. kernel, screening of quality markers and explanation of relevant action mechanism by combining UHPLC-Q-Exactive Orbitrap MS and network pharmacology.
\end{abstract}

Keywords: UHPLC-Q-Exactive Orbitrap MS, network pharmacology, stir-fried Dolichos lablab L. kernel, type-2 diabetes, molecular docking, molecular dynamics simulation, ligand channels

\section{Background}

Diabetes is directly related with pancreas which is an important component of the digestive system. Pancreas generates enzyme and hormones to help digestion of foods. Diabetes belongs to the "consumptive thirst" category in traditional Chinese medicine and it is closely related with spleen and stomach. In five elements, spleen and stomach of people are corresponding to the earth. They cover several digestive organs including pancreas of human body. According to the etiology and pathogenesis that diabetes is caused by Yin deficiency and manifested by dry heats, nitrifying spleen and Yin is the key of Yin nutrition. Radix Trichosanthis, Radix Ophiopogonis and Dolichos lablab L. which are characteristic of sweet and cool or sweet and cold are suggested as the basic medicines for symptoms of spleen and Yin deficiency. Among them, Dolichos lablab L. belongs to the spleen channel and it can 
invigorate spleen to eliminate dampness. Stir frying is equivalent to increase the strengthening effect of spleen. In traditional Chinese medicine, Dolichos lablab L. is a kind of white seeds of leguminosae plants, Dolichos L. Some study demonstrated that triterpenoid saponin has been separated from other similar plants of Dolichos L. Triterpenes and gluocosides of sterols can inhibit absorption of alcohol and sugar ${ }^{[1]}$. Although the clinical effect of stir-fried Dolichos lablab L. kernel has been approved in modern traditional Chinese medicine, existing associated studies mainly focus on its clinical studies and chemical ingredients. However, there are few studies on pharmacodynamics material basis and molecular mechanism of stir-fried Dolichos lablab L. kernel in treatment of type-2 diabetes(T2DM), thus restricting the further development and utilization of stir-fried Dolichos lablab L. kernel.

Hence, saponin chemical ingredients of stir-fried Dolichos lablab L. kernel were identified in the present study by using UHPLC-Q-Exactive Orbitrap MS technology. On this basis, active saponin ingredients related with T2DM were screened by network pharmacology. Moreover, action mechanism of screened active ingredients was interpreted. Results could provide references to screen quality markers. Molecular docking and molecular dynamics simulation were carried out based on structures of key proteins and saponin ingredients. Binding free energy was calculated and main thrust force for binding between proteins and saponin ingredients was analyzed. Besides, amino acids which play the key role in the binding between proteins and saponin ingredients were disclosed and the relevant binding mechanism was discussed. Research conclusions can provide theoretical references for development of more efficient saponin for treatment of T2DM.

\section{Experiment and Methods}

\section{Instruments and materials}

Instruments and materials used in the experiment include the series connection of four-stage rodelectrostatic field orbital hydrazine high resolution mass spectrometer and the Ultimate 3000 ultrahigh performance liquid chromatography system (Seymour Fisher Technologies Inc, USA), BT25S electronic scales (Sartorius Company), KQ-300DB numerical control ultrasonic cleaner (Kunshan Ultrasonic Instrument Co., Ltd) and methyl alcohol (Sinopharm Group Chemical Reagent Co. LTD, batch No.: 20190910).

\section{Preparation of solution samples}

Stir-fried Dolichos lablab L. kernel powder (0.5g) was taken and 10\% methyl alcohol (10ml) was added in for ultrasonic processing by $30 \mathrm{~min}$. Later, the mixture was filtered and the solution samples were gained.

\section{Chromatography and mass spectrometry conditions}

ZORBAX SB-C18 chromatographic columns $(2.1 \times 100 \mathrm{~mm}, 1.8 \mu \mathrm{m})$ were used, with a column temperature of $35^{\circ} \mathrm{C}$. The flowing phase used methyl alcohol (A)- $0.1 \%$ formic acid (B), which was 
processed by gradient elution (0-5min, 2\%A, 5-20min, 25\%-100\%A; 20-25min, 100\%-100\%A; $25-$ $27 \mathrm{~min}, 100 \%-2 \% \mathrm{~A} ; 27-30 \mathrm{~min}, 2 \%-2 \% \mathrm{~A}$ ). The flow rate and feed rate were set as $0.3 \mathrm{ml} / \mathrm{min}$ and $3 \mu \mathrm{L}$, respectively. Ultraviolet full wavelength was applied. Mass spectrometry conditions were introduced as follows: temperature of capillary tube $=320^{\circ} \mathrm{C}$, voltage of electronic spraying $=3.0 \mathrm{kv}$, flow rate of sheath gas $\left(\mathrm{N}_{2}\right)=30 \mathrm{arb}$, flow rate of auxiliary gas $\left(\mathrm{N}_{2}\right)=10 \mathrm{arbm}$ and collection range $=\mathrm{m} / \mathrm{z}$ 133-2000.

\section{Structural identification}

According to the principle that the error between testing value and theoretical value is smaller than 5ppm and nitrogen laws, the expected compounds were gained through high-resolution extraction ion chromatography by combining the Compound Discover version 3.0. MS2 data of the expected compounds was collected through the PRM mode. Finally, saponin candidates were determined according to fragment ions, retention time and references.

\section{Screening of compounds and disease targets}

3D structural files of compounds were established using chemdraw and the corresponding SMILES numbers were gained. Targets of compounds were gained through PharmMapper(http://www.lilab-ecust.cn/pharmmapper/) and SwissTargetPrediction(http:// www.swisstargetprediction.ch/) 、 TargetNet(http://targetnet.scbdd.com/calcnet/index/). The 3D structural files were submitted to PharmMapper to get targets and each compound returned 300 results. According to instructions of websites, the higher $z^{\prime}$-score reflects the higher possibility of becoming the target of compound and negative $z^{\prime}$-score represents insignificant results. Therefore, results with positive z'-score were selected. The SMILES numbers were submitted to SwissTargetPrediction and each compound returned 100 results. Since published literatures of SwissTargetPrediction have explained that the top $15 \%$ results represent $75 \%$ possibility, the top 15 returning results of each compound in SwissTargetPrediction were selected. Later, the SMILES numbers were submitted to TargetNet and results with prob higher than 0 were chosen. Results of the above three websites were summarized and used as the compound target library.

Relevant targets of type-2 diabetes were searched from CTD(http://ctdbase.org/), TTD(http://db.idrblab.net/ttd/) and GeneCards(https://www.genecards.org/),

PharmGKB(https:/www.pharmgkb.org/) with keywords of CTD: type 2 diabetes mellitus; TTD: type 2 diabetes; GeneCards: type 2 diabetes mellitus; PharmGKB: Diabetes Mellitus, Type 2. To increase reliability of data and eliminate data with low scores, GeneCards selected targets with Relevance score higher than or equal to 30. CTD chose targets with $\mathrm{M}$ or $\mathrm{T}$ (marker/mechanism|therapeutic).

Intersection between compound targets and disease targets was selected, which covered 91 genes. The Venny website is http://bioinformatics.psb.ugent.be/webtools/Venn/. 


\section{Protein interaction network}

The 91 treatment genes were input into the STRING database (https://string-db.org/) and species was limited within human. To increase reliability of results, the lowest interaction score was limited at high confidence $(0.700)$ and results were input into cytoscape3.8.0 for visualization and analysis. The network was analyzed by cytoscape plug-in and individual node parameters in the network were calculated. In this study, 22 key genes were screened by using means of Degree, BetweennessCentrality and ClosenessCentrality as the thresholds. These 22 genes might be key targets for traditional Chinese medicine treatment of type-2 diabetes.

\section{Active ingredients-target network}

Compounds corresponding to 91 therapeutic targets were identified one by one. Besides, an active ingredients-therapeutic target network was built up and input into cytoscape3.8.0 for visualization and analysis. The network was analyzed by cytoscape plug-in and individual node parameters in the network were calculated. GO and KEGG enrichment analyses of 91 therapeutic genes were finished using clusterprofiler package of Rstudio. Moreover, results were visualized by ggplot2 and put in a descending order according to pvalue. GO shows top 10 items of BP, CC and MP, respectively. KEGG shows top 20 significant pathways.

\section{Molecular docking}

Molecular docking experiment between active ingredients and key targets was carried out based on AutoDock Vina_1.1.2 to verify the interaction activity between ingredients and key targets. AutoDock Vina applied the semi-flexible molecular docking. In other words, pharmacologic molecules are flexible and rigidity of proteins is kept constant in the docking process, and docking results are evaluated by a semi-experience free energy function ${ }^{[2]}$.

\section{Molecular dynamics simulation and ligand channels}

The orbit of molecular dynamics simulation was finished by gromacs 2019-3. Graphs of root-meansquare deviation (RMSD), rotating rate, mean-square deviation (MSD), total energy, root-mean-square fluctuation (RMSF) of ligands and proteins were drawn. Clustering analysis was calculated. All potential channels for binding between ligands and proteins were explored using caver 2.0. Parameters were set as follows: minimum Probe radius $=0.9$, shell radius $=3$, and maximum shell depth in the surface region $=4$.

\section{Results and analysis}

\section{Structural identification based on UHPLC-Q-Exactive Orbitrap MS}

Ingredients of stir-fried Dolichos lablab L. kernel were identified based on the UHPLC-QExactive Orbitrap MS technology through abovementioned sample processing and analysis conditions, and then analyzed. Firstly, samples were injected into UHPLC-Q-Exactive Orbitrap MS and high- 
resolution mass data were acquired through MS full scan. Secondly, molecular formula of saponin was predicted by setting parameters of metabolic working flow for Compound Discover, and then confirmed by MS full scan and high-resolution extraction ion chromatography (HREIC). In this way, an ion list was generated. Finally, fragment ions were gained using UHPLC-Q-Exactive Orbitrap MS through parallel reaction monitoring mode (the above constructed ion list). Finally, saponin candidates were determined according to fragment ions, retention time and references. In this study, 16 saponin ingredients were identified from stir-fried Dolichos lablab L. kernel under the negative ion mode. Results are shown in Table 1. 
Table 1 Ingredient identification results of stir-fried Dolichos lablab L. kernel

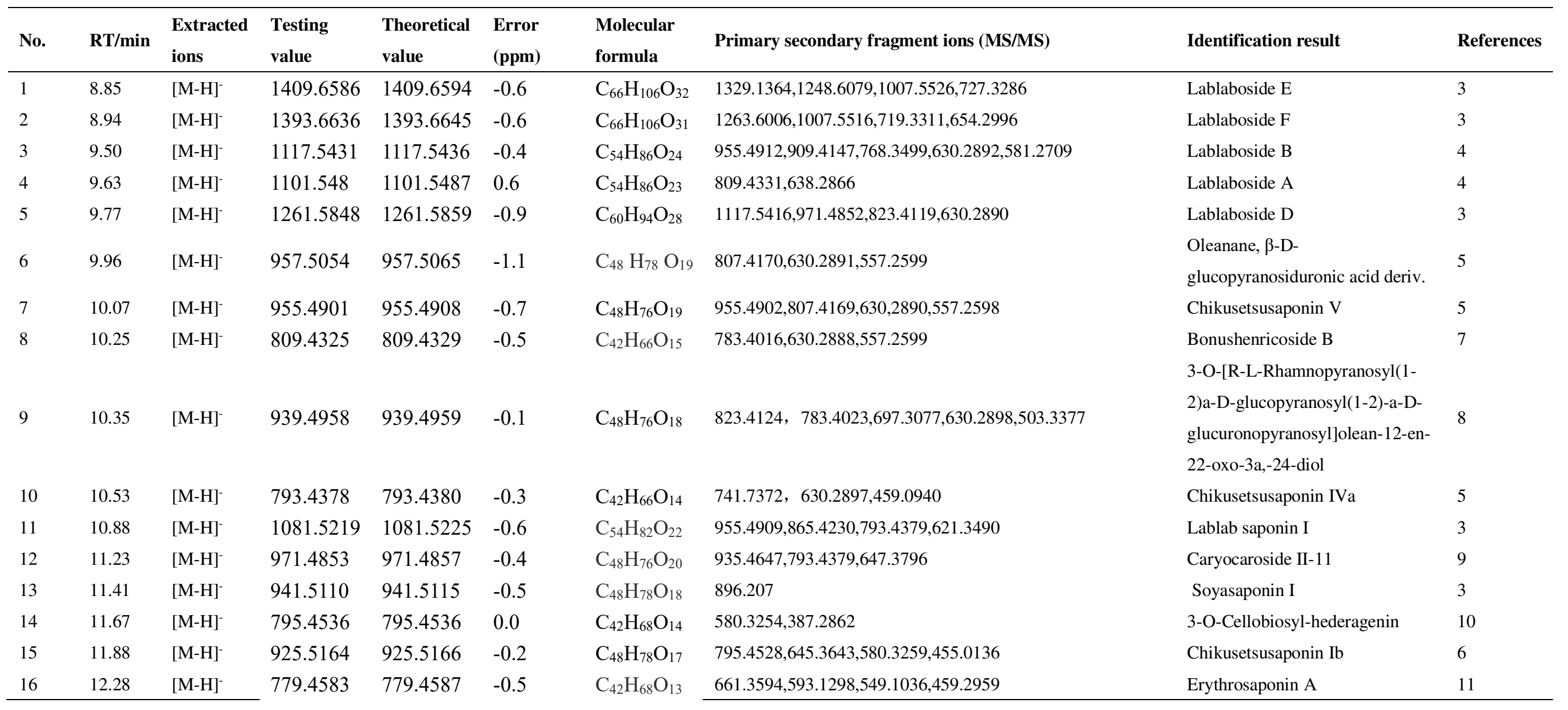


By combining with previous researches, hypoglycemic active compounds like Chikusetsusaponin Iva, Chikusetsusaponin V, Chikusetsusaponin Ib, Soyasaponin I, 3-O-Cellobiosyl-hederagenin, Oleanane, $\beta$-D-glucopyranosiduronic acid derive, lablaboside $\mathrm{B}$, Lablaboside $\mathrm{D}$, Lablaboside $\mathrm{E}$ and Lablaboside F were chosen as the representative saponin ingredients.

\section{Venn analysis of active ingredient targets and type- 2 diabetes target set}

In this study, 292 targets of saponin ingredients and 792 targets of T2DM were screened from PharmMapper, SwissTargetPrediction, TargetNet, CTD and PharmGKB databases. The 91 intersection targets for T2DM treatment based on saponin were gained through Venn analysis of saponin ingredient targets and relevant targets of T2DM.

\section{Construction and analysis of PPI network}

Based on PPI relationship, the interaction relations among therapeutic targets of T2DM based on stir-fried Dolichos lablab L. kernel were acquired from the STRING database in order to understand its action mechanism better. A total of 22 key genes were screened by using means of Degree, BetweennessCentrality and ClosenessCentrality as thresholds (Table 2). These targets are hub nodes of the network and they are core targets for treatment of T2DM.

Table 22 key genes

\begin{tabular}{llll}
\hline Gene & Degree & BetweennessCentrality & ClosenessCentrality \\
\hline STAT3 & 33 & 0.080071042 & 0.572463768 \\
MAPK1 & 32 & 0.081962504 & 0.580882353 \\
SRC & 29 & 0.056634532 & 0.530201342 \\
IGF1 & 27 & 0.050415988 & 0.533783784 \\
MAPK8 & 27 & 0.065736027 & 0.560283688 \\
JUN & 25 & 0.046517234 & 0.54109589 \\
EGFR & 25 & 0.050964745 & 0.54109589 \\
PIK3R1 & 25 & 0.050340626 & 0.506410256 \\
ALB & 23 & 0.135327763 & 0.548611111 \\
CASP3 & 20 & 0.045545357 & 0.523178808 \\
HGF & 20 & 0.033453303 & 0.506410256 \\
AR & 20 & 0.037843527 & 0.509677419 \\
ESR1 & 20 & 0.02296979 & 0.512987013 \\
MMP9 & 18 & 0.024822659 & 0.509677419 \\
NOS3 & 17 & 0.046045739 & 0.516339869 \\
SIRT1 & 17 & 0.036262484 & 0.503184713 \\
CAT & 12 & 0.065146404 & 0.475903614 \\
HMOX1 & 12 & 0.037773667 & 0.478787879 \\
F2 & 12 & 0.04549904 & 0.456647399 \\
NR3C1 & 11 & 0.037173508 & 0.454022989 \\
PPARG & 10 & 0.041550519 & 0.448863636
\end{tabular}


Four key proteins of T2DM (EGFR, IGF1, MAPK1 and PIK3R1) were chosen for molecular docking verification.

\section{GO bioprocess analysis and KEGG metabolic pathway analysis}

GO enrichment analyzes functional distribution of key targets and key targets are in a descending sequence according to pvalue. GO shows top 10 items of BP, CC and MF, respectively (Fig.1). KEGG shows the top 20 significant pathways. Additionally, the bubble graph is drawn (Fig.2). PI3K-AKT signaling pathway (Fig.3) and EGFR tyrosine kinase inhibitor resistance (Fig.4) process.

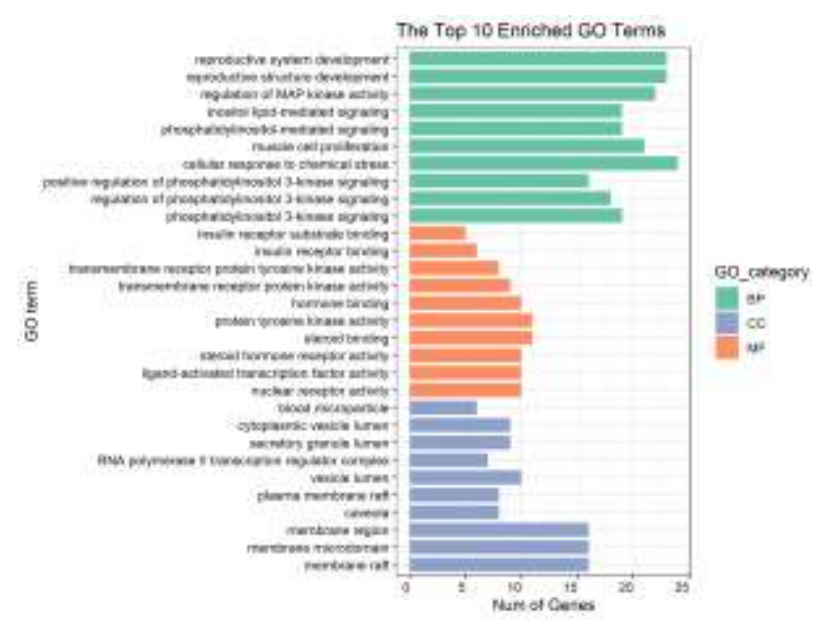

Fig.1GO enrichment analysis of main active ingredients of stir-fried Dolichos lablab L. kernel

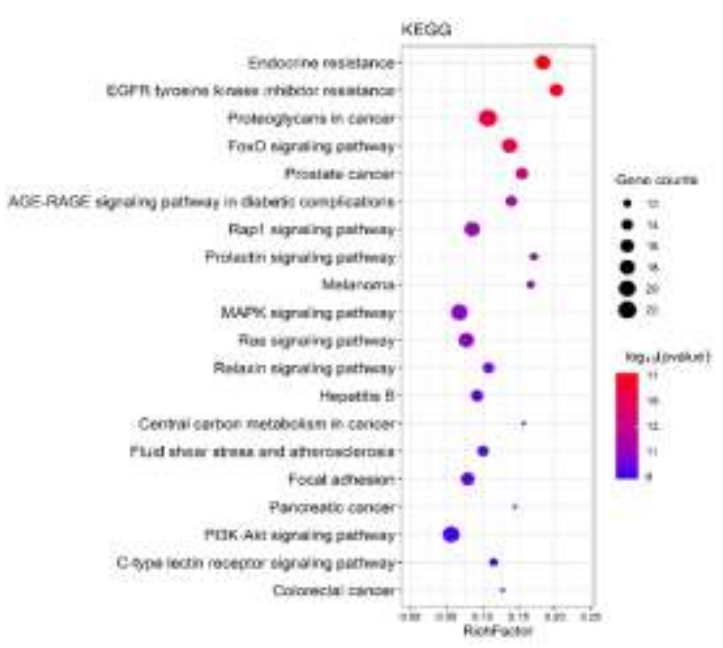

Fig. 2 KEGG bubble graph of action targets of stir-fried Dolichos lablab L. kernel on T2DM

Extracellular cytokines like HGF and IL-2 of PI3K-AKT signaling pathway can activate the downstream AKT signal pathway through PI3KR1 and PIK3CG after combined with receptors (e.g. EGFR and CSFR) on membrane. The activated AKT2 can realize phosphorylation of NOS1, RAF1, $\mathrm{BAD}, \mathrm{CHUK}$ and MDM2, thus promoting releasing of NO, activating MAPK signal pathway and facilitating generation of BCL2L1 and BCL2. These can influence apoptosis and NF- $\kappa$ B cell pathways, regulate effects of TP53 on p53 signal pathway, and control cell proliferation, angiogenesis, DNA 
repair and cell survival.

The PIK3R1/AKT signal shaft, JAK/STAT3, SRC/STAT3 signal shaft and MAPK signal shaft are activated by combinations of EFG, TGFA, HGF, IGF1,VEGFA and FGF with receptors on membrane like EGFR, ERBB2, ERBB3, MET, IGF1R, KDR and FGFR2. AKT2 can promote phosphorylation of MTOR, BAD, GSK3B and FOXO3. Phosphorylation of MAPK1 can inhibit expression of BAX by promoting expression of BCL2L1 and BCL, thus inhibiting apoptosis. The phosphorylated FOXO3, STAT3 and MAPK1 can develop the transcriptional control effect after entering into nucleus. All of these downstream molecules regulate differentiation, growth, proliferation, survival and activity of cells as well as angiogenesis.

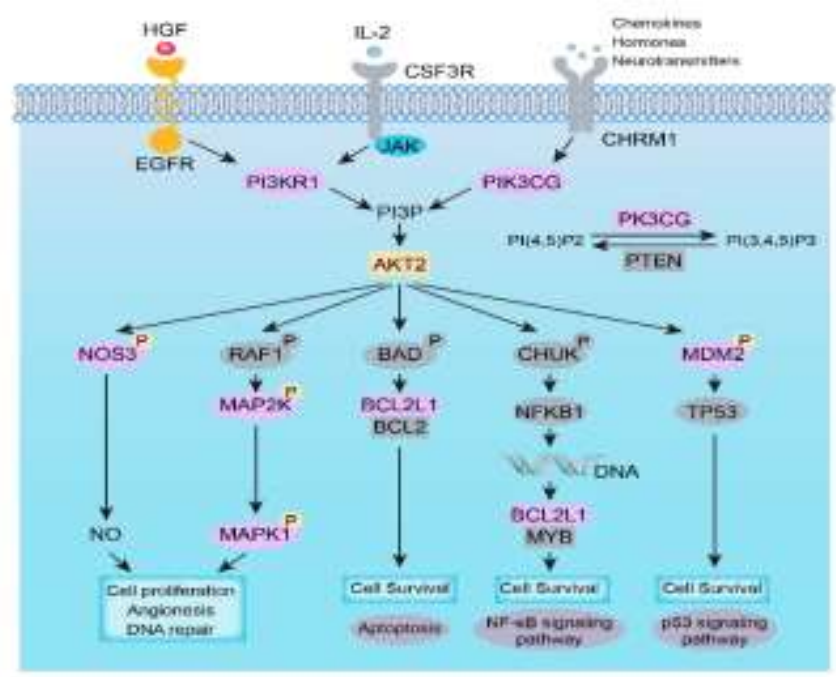

Fig.3 PI3K-AKT signaling pathway

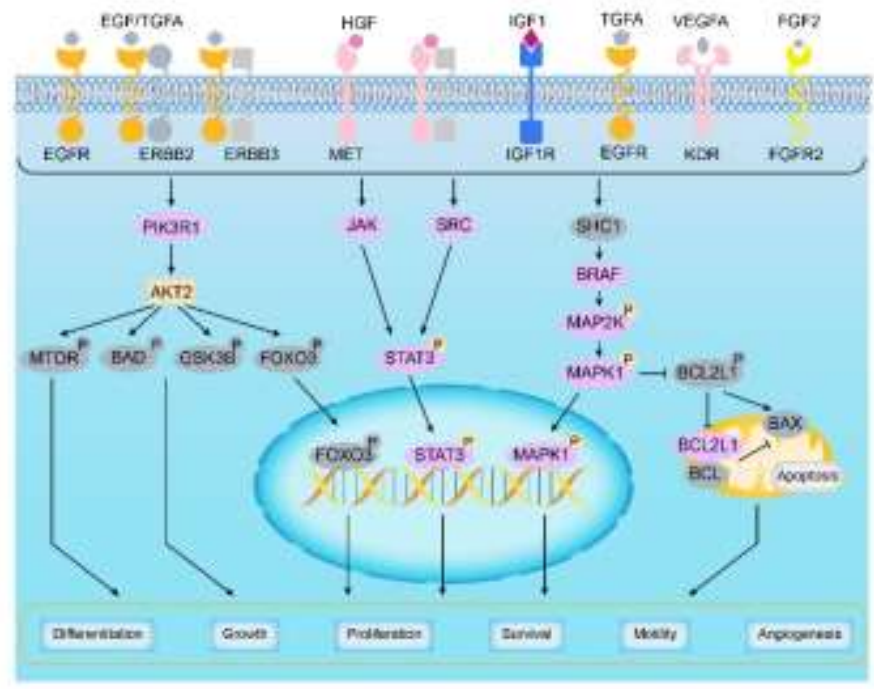

Fig.4 EGFR tyrosine kinase inhibitor resistance

\section{Building of an active ingredients-targets-pathway network}

The Saponin active ingredients-targets-signal pathway network was built using Cytoscape3.8.0 
(Fig.5). It can be seen from Fig.5 that there are 101 nodes (10 compounds and 91 targets) and 593 sides. The V-shaped nodes represent compounds, while the round nodes represent genes. Colors of nodes from light to dark represent the increasing values of node degree. The therapeutic mechanism of stir-fried Dolichos lablab L. kernelsaponin ingredients to T2DM is related with inflammatory response, autoimmune damage and several other morbidity theories. This study interpreted the therapeutic mechanism of stir-fried Dolichos lablab L. kernelsaponin ingredients to T2DM from different perspectives.

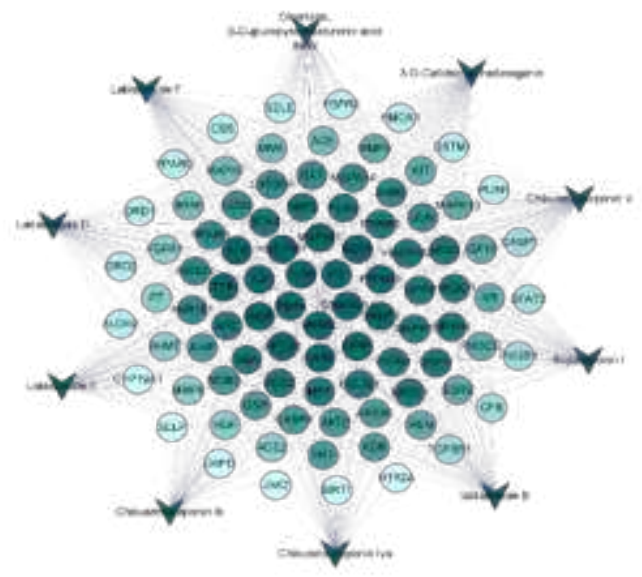

Fig.5 Saponin active ingredients-targets-signal pathway network

\section{Molecular docking}

Molecular docking experiment between 10 saponin active ingredients and the screened key protein targets related with T2DM was carried out using AutoDock Vina_1.1.2. AutoDock Vina evaluates the bonding strength between micromolecules and proteins mainly through affinity, that is, the fitted $\Delta \mathrm{G}$ value after calculation. If $\Delta \mathrm{G}$ value is lower than 0 , the ligands can bind with receptors spontaneously. Moreover, the lower $\Delta \mathrm{G}$ value brings the higher affinity and the active ingredients are easier to bind with receptors ${ }^{[12]}$. Results are listed in Table 3. Graphs are drawn for each protein and the best docked compound in Table 3 (Fig.6). Clearly, lablaboside_E enters into the active pockets of EGFR completely and forms one or two hydrogen bonds with active site residues Thr830, Cys773, Lys721, Asp831, Arg817, Val852, Ile854 and Lys851, thus forming 12 hydrogen bonds. Additionally, lablaboside_E not only forms salt bridges with residues Lys855 and Lys851, but also forms Van Der Waals' force with residues Asp776, Asn818, Gly772, Arg779, Lys889, Leu834, Leu838, Phe699, Ser696, Ala698, Trp856 and Ala896. It also forms alkyl interactions with residues Ala719, Leu820, Val702, Leu775 and Leu694. The docking results reflect that lablaboside_F is bonded on the surface of IGF1 and forms three hydrogen bonds with active sites. These hydrogen bonds involve residues Asp12, Glu9 and Cys6, respectively. Lablaboside_F forms alkyl interactions with residues Ala13, Leu5, Leu57, Leu54 and Val11, and forms Van Der Waals' force with residues Phe16, Gln15, Glu3 and Phe23. 
According to docking results, lablaboside_F enters into the active pocket of MAPK1 and forms 14 hydrogen bonds with active site residues, mainly involving Gln105, Lys54, Asp167, Asn154, Tyr36, Ala35, Val188, Thr190, Trp192 and Arg191. Residues Val39, Ile31, Tyr113 and Tyr30 participate in the formation of interactions with alkyl groups or pi-alkyl groups, while residues Ile84, Leu156, Thr110, Ala52, Cys166, Lys114, Gly230, Ala189, Asp149, Leu170, Gly34, Ser153, Glu33and Asp111 form Van Der Waals' force with lablaboside_F. It can be seen from Fig.6 that chikusetsusaponin_Iva enters into active site of PIK3R1 completely. It forms not only four hydrogen bonds with residues Arg1818, Gln1815, Gln1630 and Glu1849, but also Van Der Waals' force with residues Arg1852, Arg1281, Ser1275, Leu1279, His1759, Asn1756, Phe1794, Ile163, Met1811, His1670, Tyr1836, Pro1835, Gly1837 and Leu1755. Chikusetsusaponin_Iva also forms alkyl interactions with residues Leu1834 and Val1851.

Table 3 Molecular docking affinity between saponin and key proteins

\begin{tabular}{lrrrr}
\hline COMPOUND & EGFR & \multicolumn{1}{c}{ IGF1 } & MAPK1 & PIK3R1 \\
\hline Lablaboside_F & -10 & -7.6 & -10.2 & -9.5 \\
Lablaboside_E & -10.7 & -7.3 & -10.2 & -8.8 \\
3-O-Cellobiosyl-hederagenin & -9.3 & -6.8 & -9.8 & -9.5 \\
Chikusetsusaponin_Ib & -9.7 & -6.4 & -9.7 & -8.7 \\
Oleanane & -8.8 & -6.7 & -9.7 & -8.6 \\
Soyasaponin_I & -10.1 & -7.2 & -9.5 & -9.9 \\
Chikusetsusaponin_V & -9.7 & -6.9 & -9.5 & -8.7 \\
Chikusetsusaponin_Iva & -10 & -7 & -9.4 & -10.3 \\
Lablaboside_D & -10.2 & -5.7 & -9.3 & -7.8 \\
lablaboside_B & -9.1 & -6.3 & -8.9 & -7.2 \\
\hline
\end{tabular}
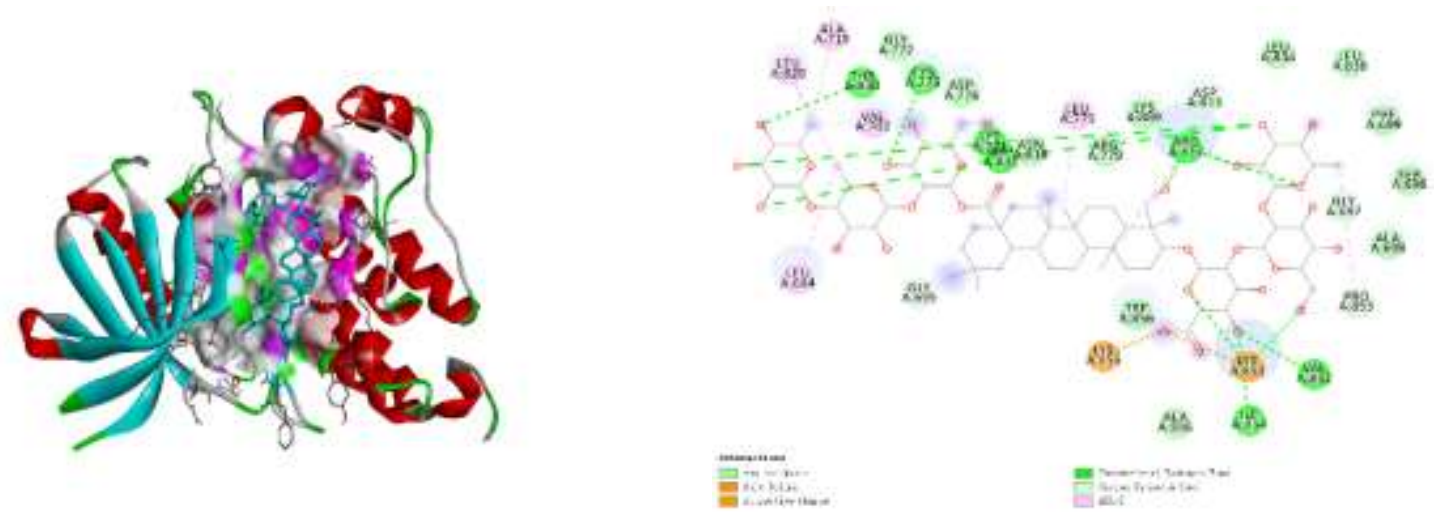

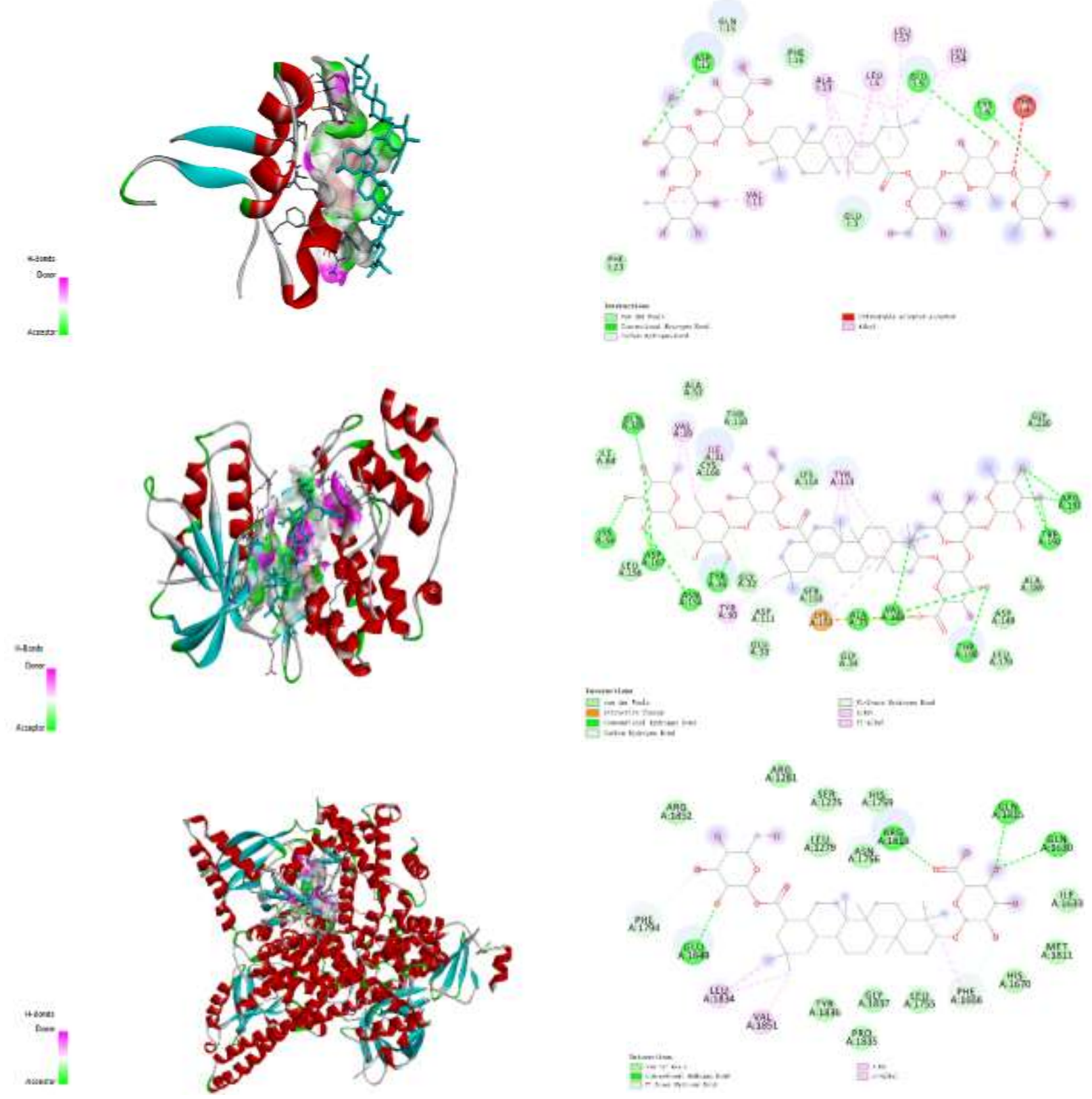

Fig.6 Docking mode analysis between the lowest-affinity ingredients and targets

\section{Molecular dynamics simulation and ligand channels}

According to molecular docking results, molecular dynamics simulation on Chikusetsusaponin Iva which has relatively binding with the key protein PIK3R1 was carried out, which further verified stability and reasonability of docking results. The trajectory of protein rotating value is shown in Fig.7. This trajectory is used to study the mean distance of each protein atom to the center, thus illustrating closeness of each corresponding proteins. The trajectory of protein MSD is used to calculate the error between each ligand and protein. 

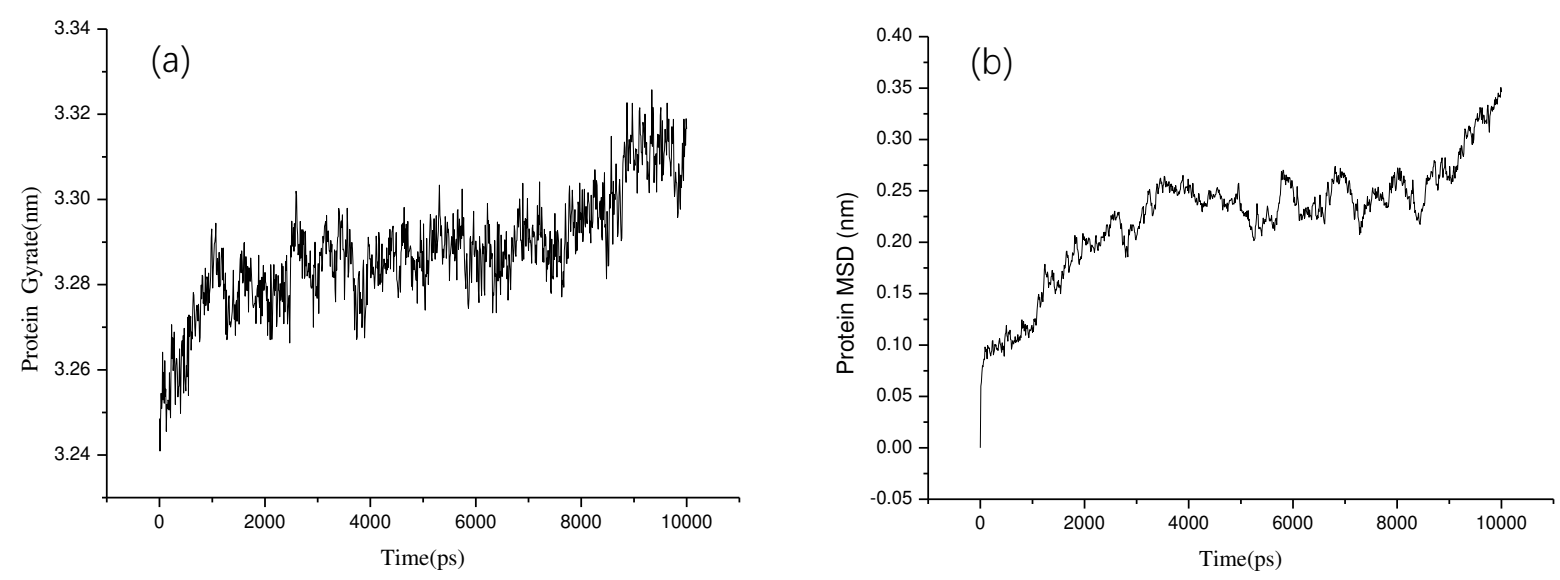

Fig.7 (a) Protein rotating value (b) MSD of proteins

The total energy trajectory graph can be used to evaluate stability of ligand-protein compound. The mean potential energy, kinetic energy and total energy which are needed for ligand-protein binding were calculated as $-2923388.5 \mathrm{KJ} / \mathrm{mol}, 552577.6 \mathrm{KJ} / \mathrm{mol}$ and $-2370811 \mathrm{KJ} / \mathrm{mol}$, respectively. Based on these parameters, binding stability of ligand-protein compounds was compared.

Representative structure of the ligand-protein compounds was discussed through clustering analysis. There are 7 classes of typical structures of Chikusetsusaponin Iva-protein compounds (Fig.8).
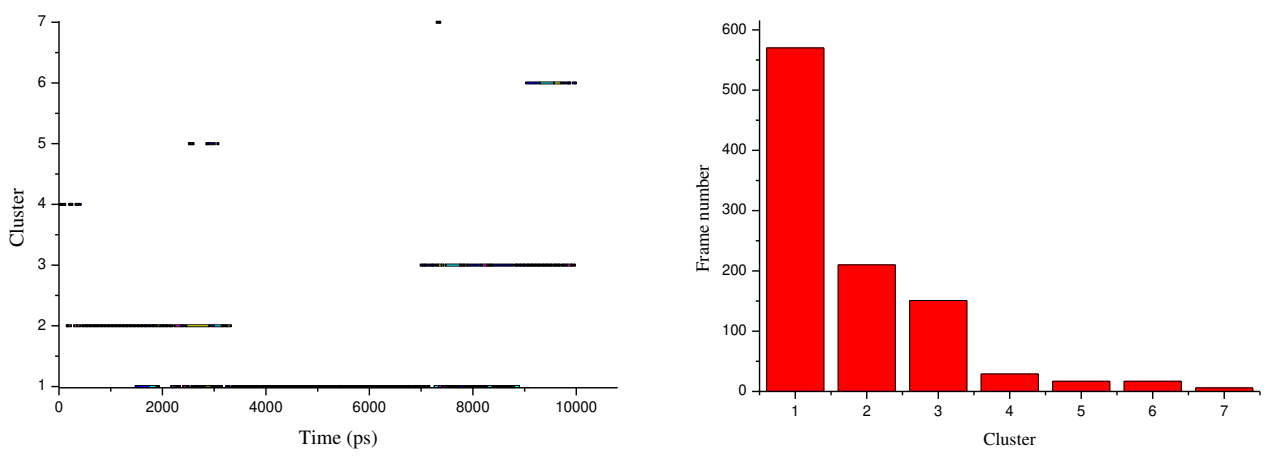

Fig. 8 Clustering analysis

Three-dimensional simulation of ligand channels was constructed to estimate all possible channels for combination of ligands and proteins. Chikusetsusaponin Iva has 15 possible pathways (Fig.9). 


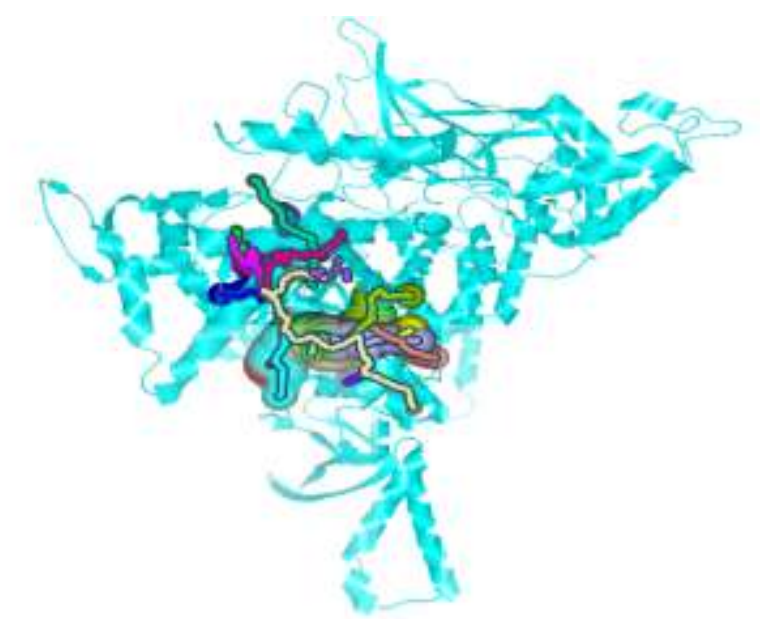

Fig.9 Three-dimensional simulation of ligand channels

\section{Discussions}

Four key proteins, including EGFR, IGF1, MAPK1 and PIK3R1, were chosen. Chu et al. ${ }^{[13]}$ discovered that systematic insulin-like growth factor-1(IGF1-1) therapy can reverse the hyperpathia phenomena of mice with diabetic peripheral neuropathy and improve their mobility. Mauvaris Jarvis ${ }^{[14]}$ pointed that PI3 kinase plays the crucial role in metabolism of insulin. Moreover, the levels of p85 $\alpha$ and AS53 could be decreased by hybrid damages to PIK3R1 genes, thus enabling to increase insulin sensitivity and lower fasting and postprandial blood glucose levels. As result, morbidity of diabetes which is related with genetic insulin resistance is decreased significantly. MAPK3 and MAPK1 are two crucial MAPKs in MAPK/ERK cascading. MAPK/ERK pathway plays an important role in diabetes and its complications. It is reported that blocking EGFR can inhibit infiltration and oxidative stress of kidney immune cells and strengthen autophagy activity of pancreas, finally improving diabetic nephropathy ${ }^{[15]}$.

Compounds (Lablaboside E, Lablaboside F, Chikusetsu saponin Iva and Chikusetsu saponin Iva) which have strong bindings with 4 key proteins can protect hyperglycemia-induced INS-1cells from functional damages. The possible molecular mechanisms might be attributed to the anti-apoptosis effect by activating the Akt/mTOR signal pathway. Mixtures of glucosides were gained through separation of seeds of Dolichos lablab L.. These mixtures possess outstanding activity of immunologic adjuvants. Six oleanane-type bissaponin compounds which are separated and identified from these glucoside mixtures with activity of immunologic adjuvants are named Lablaboside A, B, C, D, E and F. Lablaboside A, B, C, D, E and F not only have immunological enhancement, but also can lower blood glucose ${ }^{[1]}$. The blood glucose lowering mechanism might be related with improving immunological functions of patients with diabetes, adjusting combination of insulin and receptors, and increasing sensitivity of body to insulin.

According to metabolic pathway analysis, EGFR signal pathway is the pathway with a relatively 
high enrichment factor. Pathways which have relatively more enrichment targets include PI3K-Akt signal pathway, MAPK signal pathway, Ras signal pathway, Rap1 signal pathway, and pathways of proteoglycans in cancer. Some studies demonstrate that therapeutic pathways of T2DM based on saponin of stir-fried Dolichos lablab L. kernel include cancer pathways like proteoglycans in cancer. According to a large-scaled epidemiological investigation, morbidity of cancers at some specific positions in patients with $\mathrm{T} 2 \mathrm{DM}$ is significantly higher compared to that of other patients ${ }^{[16]}$. In addition, cancer mortality of patients with T2DM is relatively higher. The mechanism can be interpreted by that the effect of hyperinsulinemia on microenvironment of cancer cells and signal transduction in cells of diabetic patients is in favor of tumor growth ${ }^{[17]}$. Rap which belongs to Ras family has two subtypes: Rap1 and Rap2. Rap controls many important signal pathways in cells. On one hand, these pathways are closely related with important biological functions, such as formation of cell polarity, cell proliferation, differentiation and canceration, cell adhesion and movement. On the other hand, they further affect some important physiological functions of tissues and organs, such as establishing of nerve polarity, synaptic growth, synaptic plasticity, neuronal migration, and so on.

GO analysis finds that main enrichment entries of molecular functions include steroid hormone receptor activity, steroid binding, protein tyrosine kinase activity, insulin receptor binding and hormone binding. Main enrichment entries in the biological process (BP) include cellular response to chemical stress, regulation of MAP kinase activity and reproductive system development. Main enrichment entries of cellular component (CC) mainly distribute in membrane region, vesicle lumen and membrane microdomain. According to GO analysis, therapeutic mechanism of T2DM based on saponin of stir-fried Dolichos lablab L. kernel might lie in the binding between insulin and specificity insulin receptor on plasma membrane. Defects of receptors can influence the downstream signal network. Insulin receptor level covers the affinity between insulin and insulin receptor as well as the insulin receptor tyrosine kinase (IRTK).

It can be seen from molecular docking experiment that all compounds can enter into the active sites of targets and form 3-14 hydrogen bonds with active site residues. Moreover, Van der Waals' force exists universally between compounds and active sites. According to affinity, all compounds form strong binding with targets, indicating that compounds can treat T2DM by adjusting activity of these targets.

\section{Conclusion}

Based on acquisition of accurate chemical ingredients, active ingredients of stir-fried Dolichos lablab L. kernel for treatment of T2DM and relevant therapeutic mechanism are discussed in this study by combining UHPLC-Q-Exactive Orbitrap MS analysis and network pharmacology. Research conclusions lay a solid foundation for identification of effective ingredients, screening of mass markers 
and interpretation of action mechanism of stir-fried Dolichos lablab L. kernel.

Availability of data and materials

The datasets used and/or analysed during the current study are available from the corresponding author on reasonable request

\section{Abbreviations}

T2DM: type-2 diabetes

\section{Acknowledgements}

Not applicable.

\section{Funding}

Not applicable.

\section{Author information}

\section{Affiliations}

Beijing Tcmages Pharmaceutical Co., LTD, Beijing, P.R. China

Jun Han

\section{Hunan Academy of Chinese Medicine, Hunan University of Chinese Medicine, Changsha, P.R. China} Liangzi Fang

Key Laboratory of Dong Medical Research of Hunan Province, Hunan University of Medicine, Huaihua, P.R. China

Qinfang Zheng

\section{Contributions}

$\mathrm{J} \mathrm{H}$ responsible in the study design and experiments and wrote the manuscript. LZF and QFZ carried out the experiments and MS analysis. All authors read and approved the final manuscript.

\section{Corresponding authors}

Correspondence to Jun Han

\section{Ethics declarations}

Ethics approval and consent to participate

Not applicable.

\section{Consent for publication}

Not applicable.

\section{Competing interests}

The authors declare that they have no competing interests.

\section{References:}

[1] Yang Zhongyi. High-efficiency synthesis of Lablaboside F [D]. Zhengzhou University, 2013.

[2] Xu Hongbin, Li Peixing, Qi Xiangjun et al. Research on therapeutic mechanism of Shufeng detoxification capsule in COVID-19 based on network pharmacology and molecular docking [J/OL]. Chinese Medicinal Materials, 2020(08): 2056-2064[2020-1014].https://doi.org/10.13863/j.issn1001-4454.2020.08.049. 
[3]Ha T J, Lee B W, Park K H, et al. Rapid characterisation and comparison of saponin profiles in the seeds of Korean Leguminous species using ultra performance liquid chromatography with photodiode array detector and electrospray ionisation/mass spectrometry (UPLC-PDA-ESI/MS) analysis[J]. Food Chemistry, 2014, 146(mar.1):270-277.

[4]Yoshikawa M, Murakami T, Komatsu H, et al. Medicinal foodstuffs. XII. Saponin constituents with adjuvant activity from hyacinth bean, the seeds of Dolichos lablab L. (1): Structures of lablabosides A, B, and C.[J]. Chemical \& Pharmaceutical Bulletin, 1998, 29(5): 812-816.

[5]Shi J, Cai Z, Chen S, et al. Qualitative and quantitative analysis of saponins in the flower bud of Panax ginseng (Ginseng Flos) by UFLCmil riple TOF cm S/MS and UFLCKMTRAP cm S/MS[J]. Phytochemical Analysis, 2019, 31(3).

[6] Chen Jiali, Tan Mengxia, Zou Lisi, et al. Qualitative and Quantitative Analysis of the Saponins in Panacis Japonici Rhizoma Using Ultra-Fast Liquid Chromatography Coupled with Triple Quadrupole-Time of Flight Tandem Mass Spectrometry and Ultra-Fast Liquid Chromatography Coupled with Triple Quadrupole-Linear Ion Trap Tandem Mass Spectrometry[J].Chemical \& Pharmaceutical Bulletin, 2019, 67(8):839-848.

[7]Kokanova-Nedialkova Z, Nedialkov P T, Momekov G. Saponins from the roots of Chenopodium bonus-henricus L.[J]. Natural Product Letters.

[8]Balestri F, De Leo M, Sorce C, et al. Soyasaponins from Zolfino bean as aldose reductase differential inhibitors[J]. Journal of Enzyme Inhibition \& Medicinal Chemistry, 2019, 34(1):350360.

[9]Magid A A, Voutquenne L, Moretti C, et al. Triterpenoid Saponins from the Fruits of Caryocar glabrum[J]. Journal of Natural Products, 2006.

[10]Zhang F X , Li M , Yao Z H , et al. A target and non-target strategy for identification or characterization of the chemical ingredients in Chinese herb preparation Shuang-Huang-Lian oral liquid by ultra-performance liquid chromatography-quadrupole time-of-flight mass spectrometry.[J]. Biomedical Chromatography, 2017:e4110.

[11]Kaennakam S, Aree T, Yahuafai J, et al. Erythrosaponins A-J, triterpene saponins from the roots and stem bark of Gardenia erythroclada[J]. Phytochemistry, 2018, 152:36-44.

[12] Xu Hongbin, Qi Xiangjun, Fang Caishan et al. Research on antitumor mechanism of andrographolide based on network pharmacology and molecular docking [J]. Chinese Journal of Hospital Pharmacy, 2020, 40(12): 1312-1319.

[13]Chu Q, Moreland R, Yew N S, et al. Systemic insulin-like growth factor-1 reverses hypoalgesia and improves mobility in a mouse model of diabetic peripheral neuropathy $[\mathrm{J}]$. Mol Ther, 2008, 16( 8) :1400-1408.

[14]Mauvais-Jarvis F, Ueki K, Fruman D A, et al. Reduced expression of the murine p85 $\alpha$ subunit of phosphoinositide 3-kinase improves insulin signaling and ameliorates diabetes $[\mathrm{J}]$. J Clin Invest, 2002, 109( 1) : 141-149.

[15]LI Zhilian, LI Yan, OVERSTEET J M, et al. Inhibition of epidermal growth factor receptor activation is associated with improved diabetic nephropathy and insulin resistance in type 2 diabetes [J] .Diabetes, 2018, 67( 9) : 1847-1857.

[16]GIOVANNUCCIE, HARLANDM, ARCHER M C, et al. Diabetes and cancer: a consensus report [J] . Diabetes Care, 2010, 33( 7) : 1674-1685.

[17] Xu Jiana, Li Bingbing, Chen Zhenlin, et al. Network pharmacology research of total saponins of 
ginseng in treatment of type-2 diabetes [J]. Journal of Guangdong Pharmaceutical University, 2020, 36(01): 50-59. 


\section{Figures}

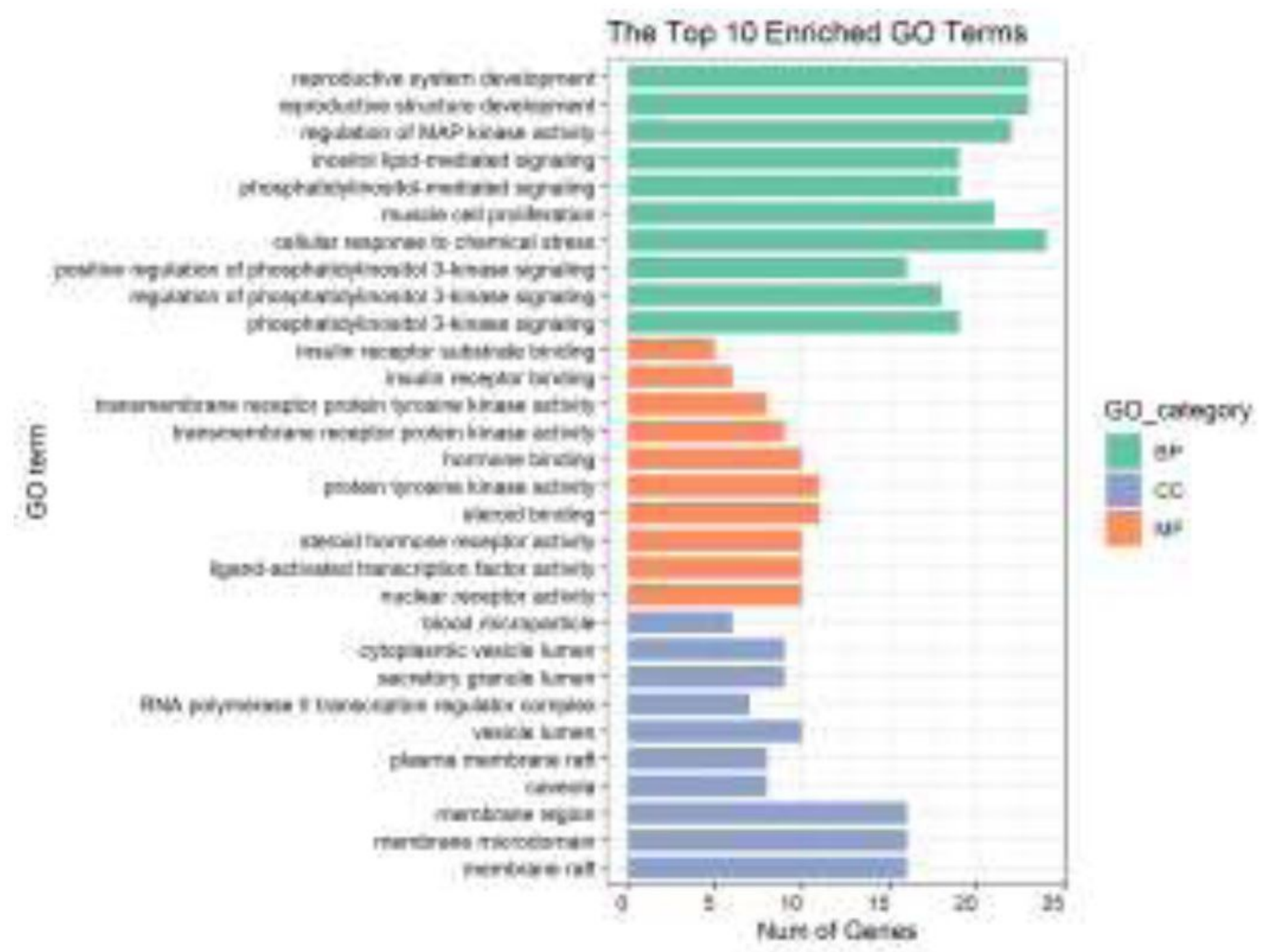

Figure 1

GO enrichment analysis of main active ingredients of stir-fried Dolichos lablab L. kernel 


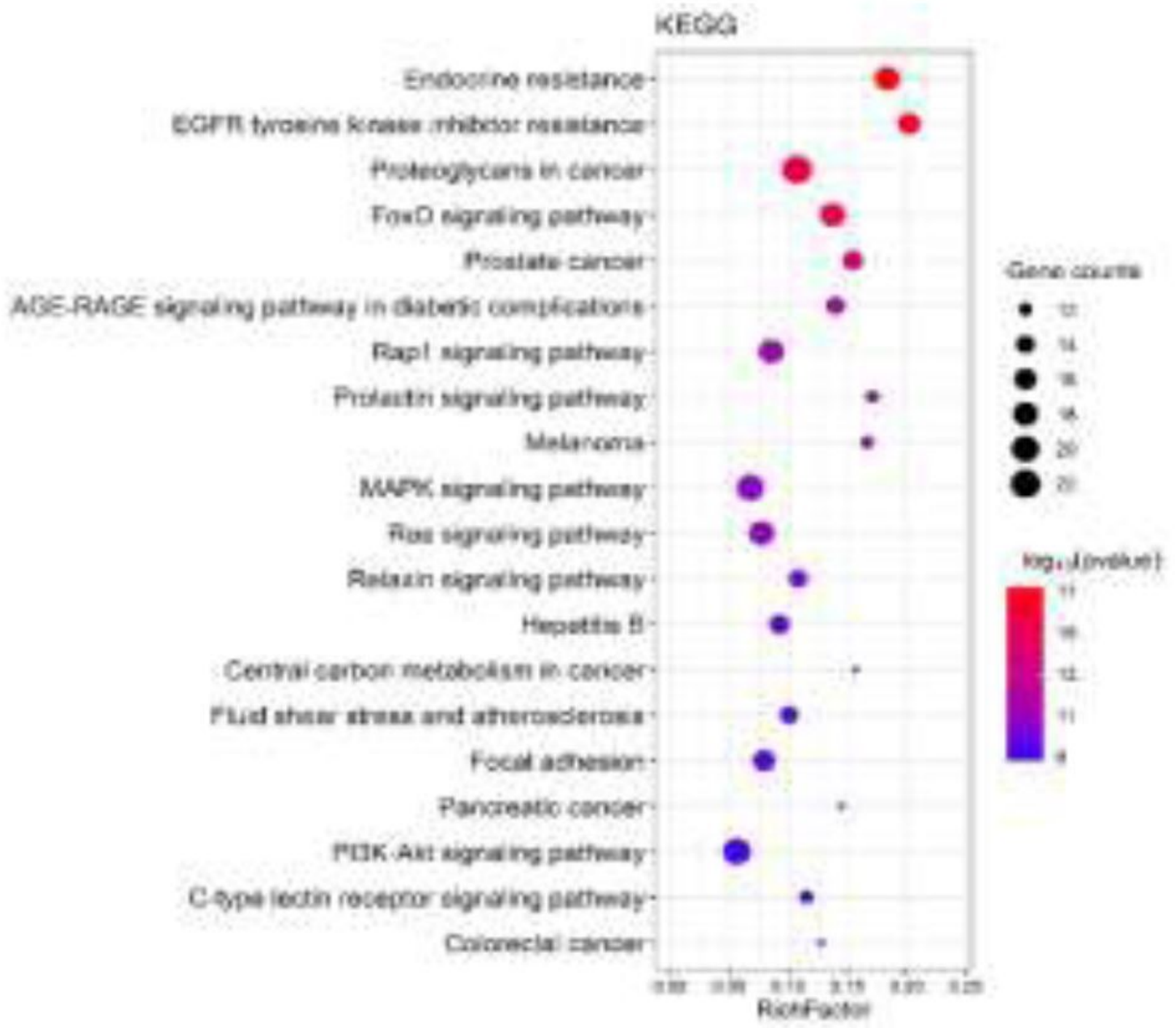

Figure 2

KEGG bubble graph of action targets of stir-fried Dolichos lablab L. kernel on T2DM 


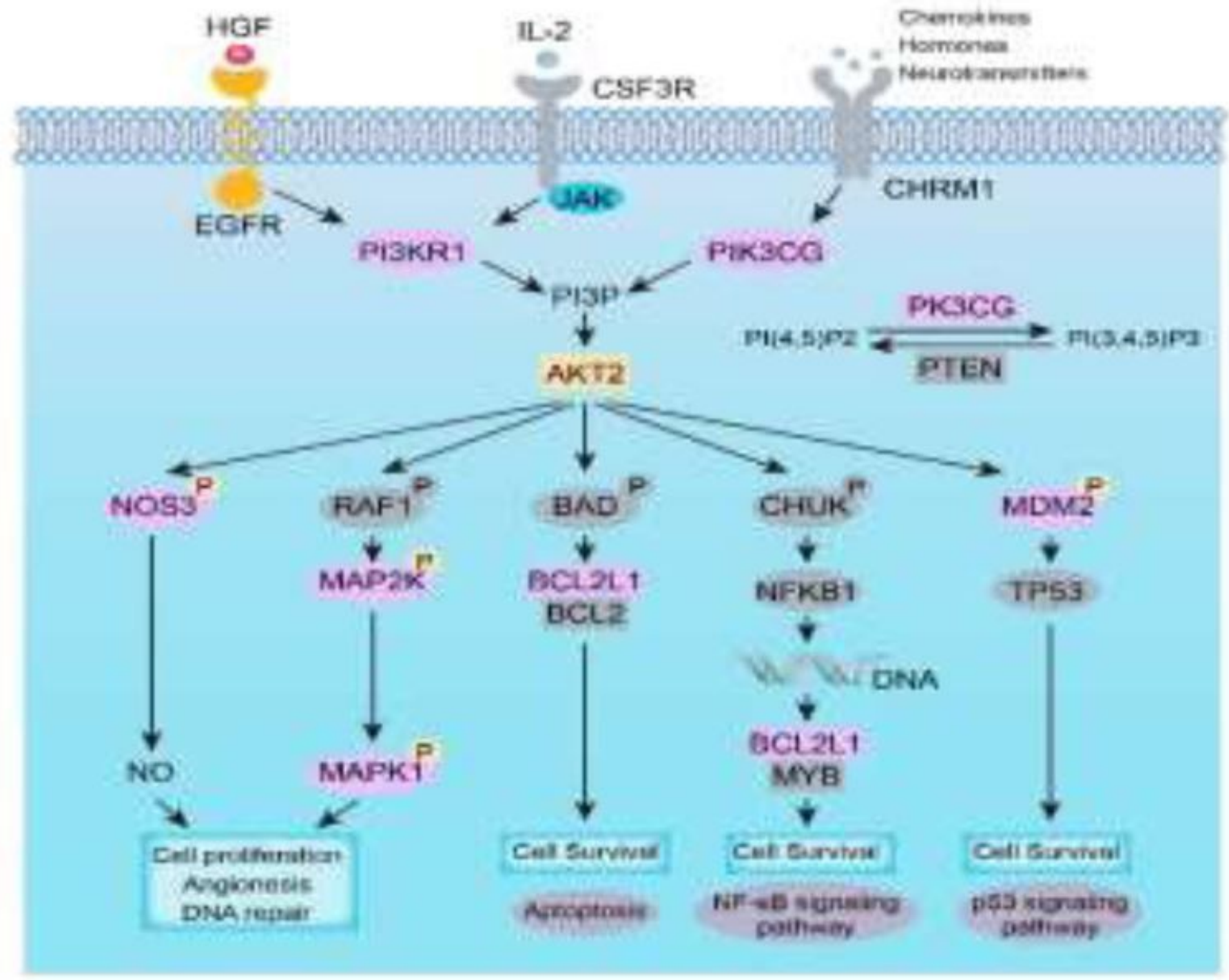

Figure 3

PI3K-AKT signaling pathway 


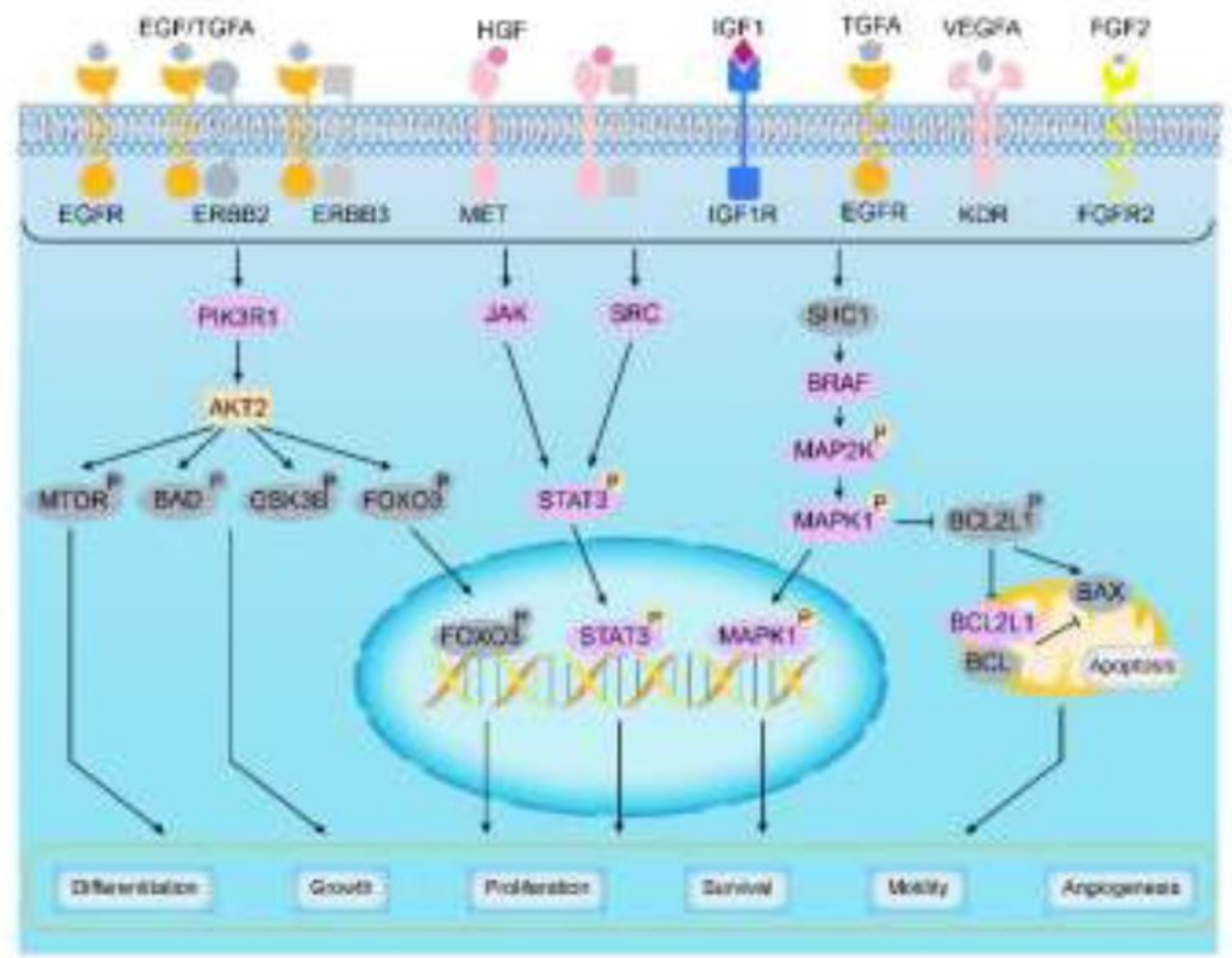

Figure 4

EGFR tyrosine kinase inhibitor resistance 


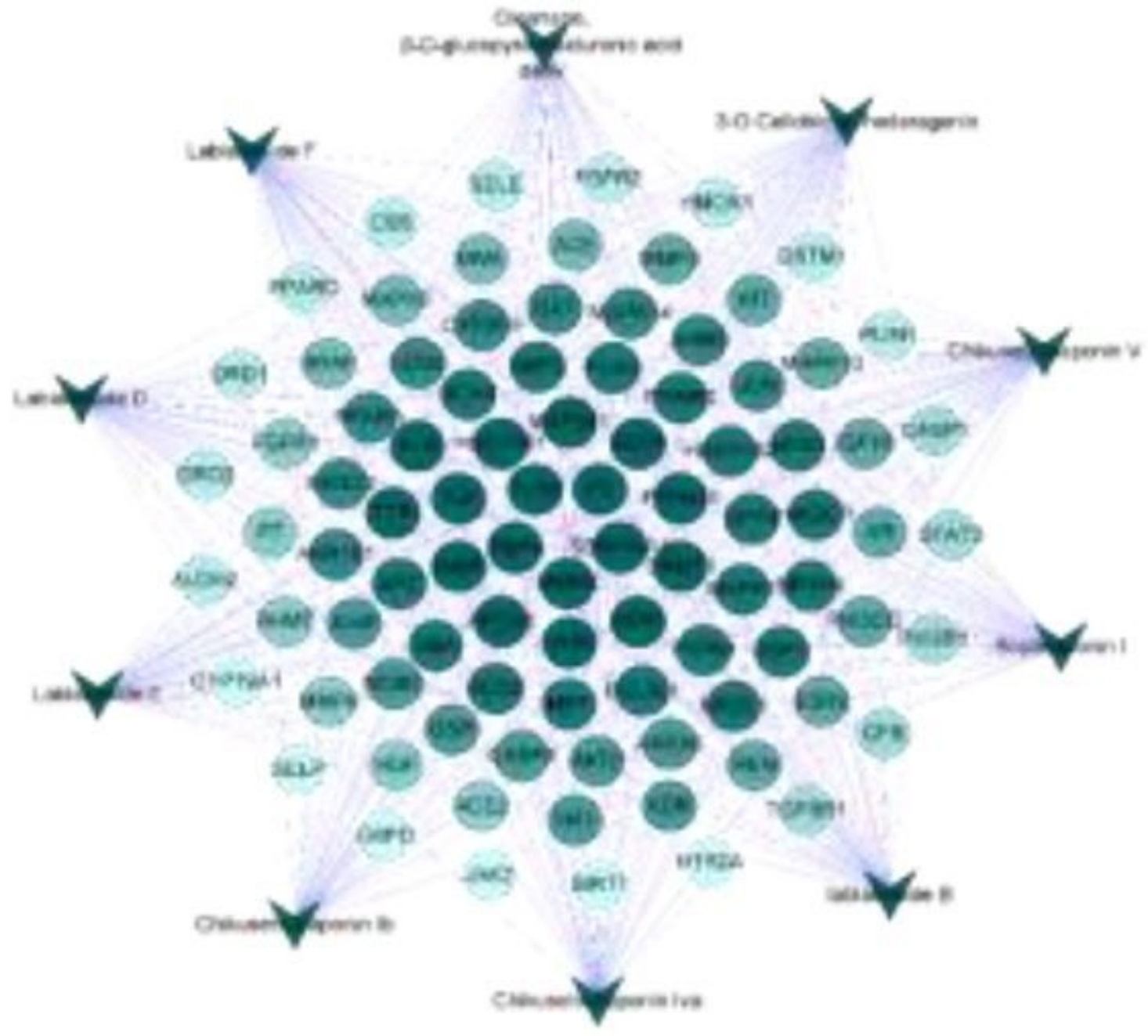

Figure 5

Saponin active ingredients-targets-signal pathway network 

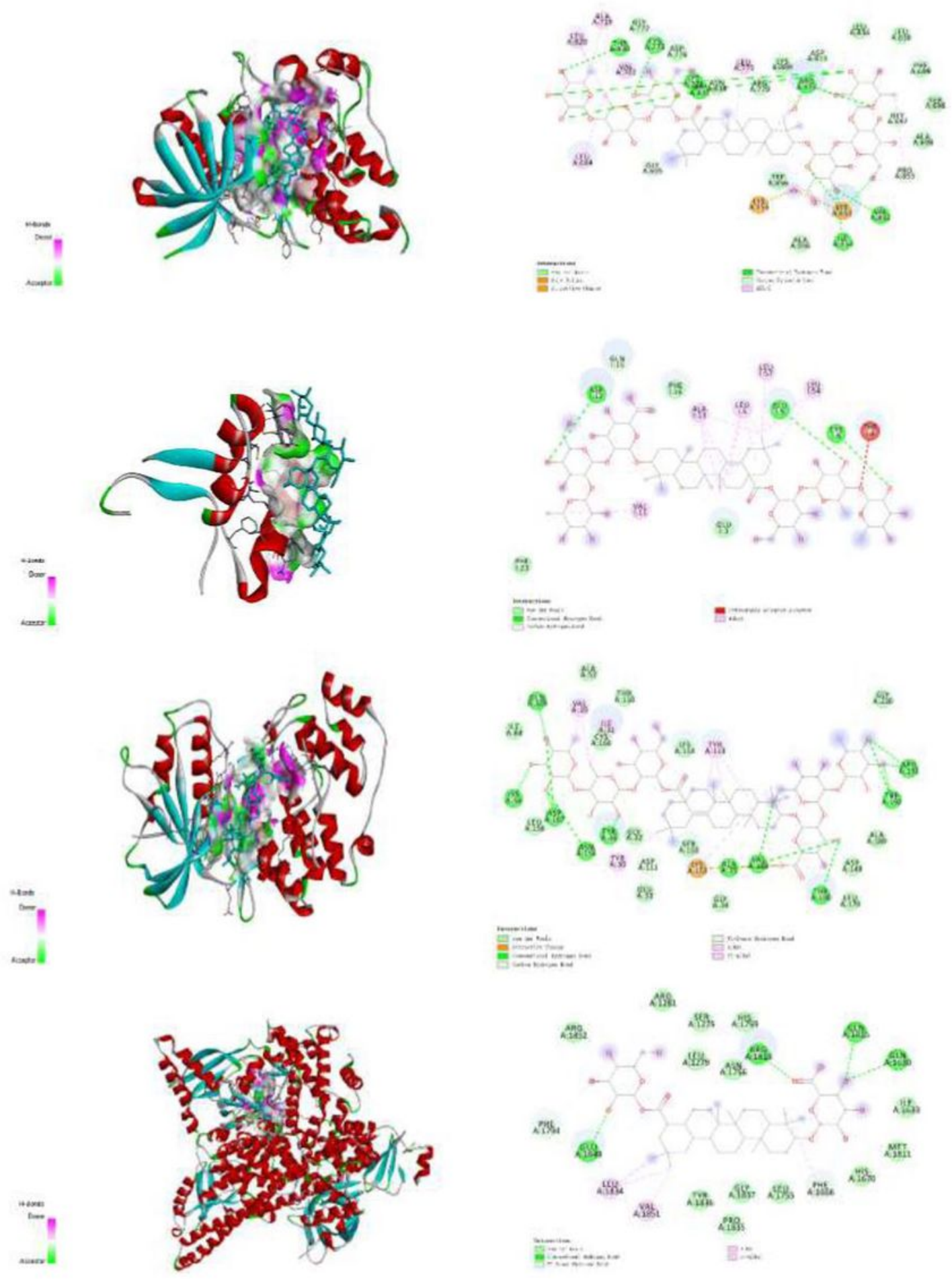

\section{Figure 6}

Docking mode analysis between the lowest-affinity ingredients and targets 

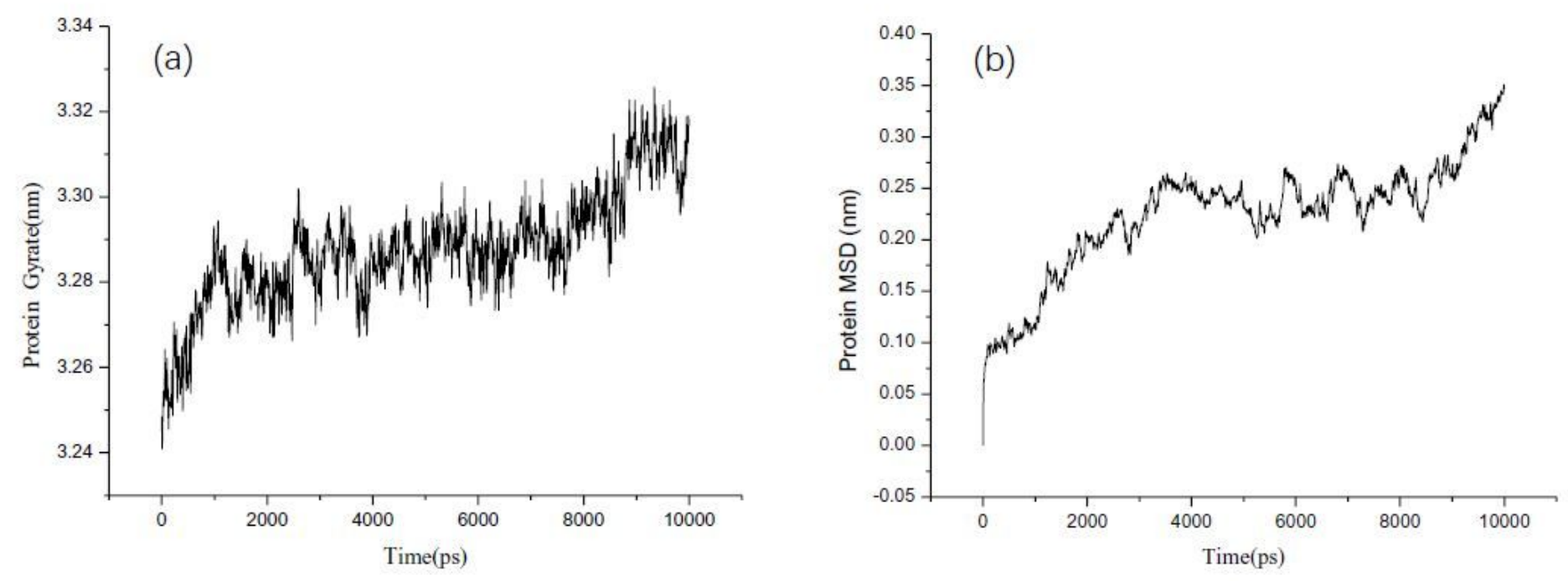

Figure 7

(a) Protein rotating value (b) MSD of proteins
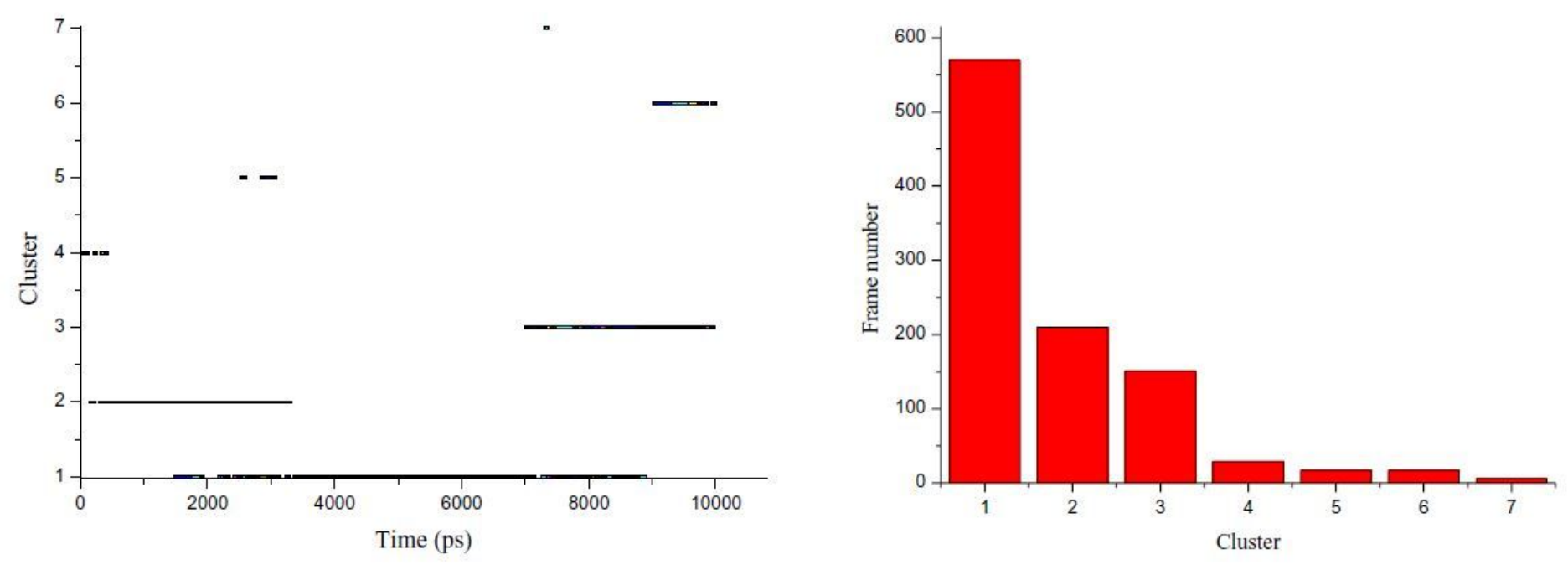

Figure 8

Clustering analysis 


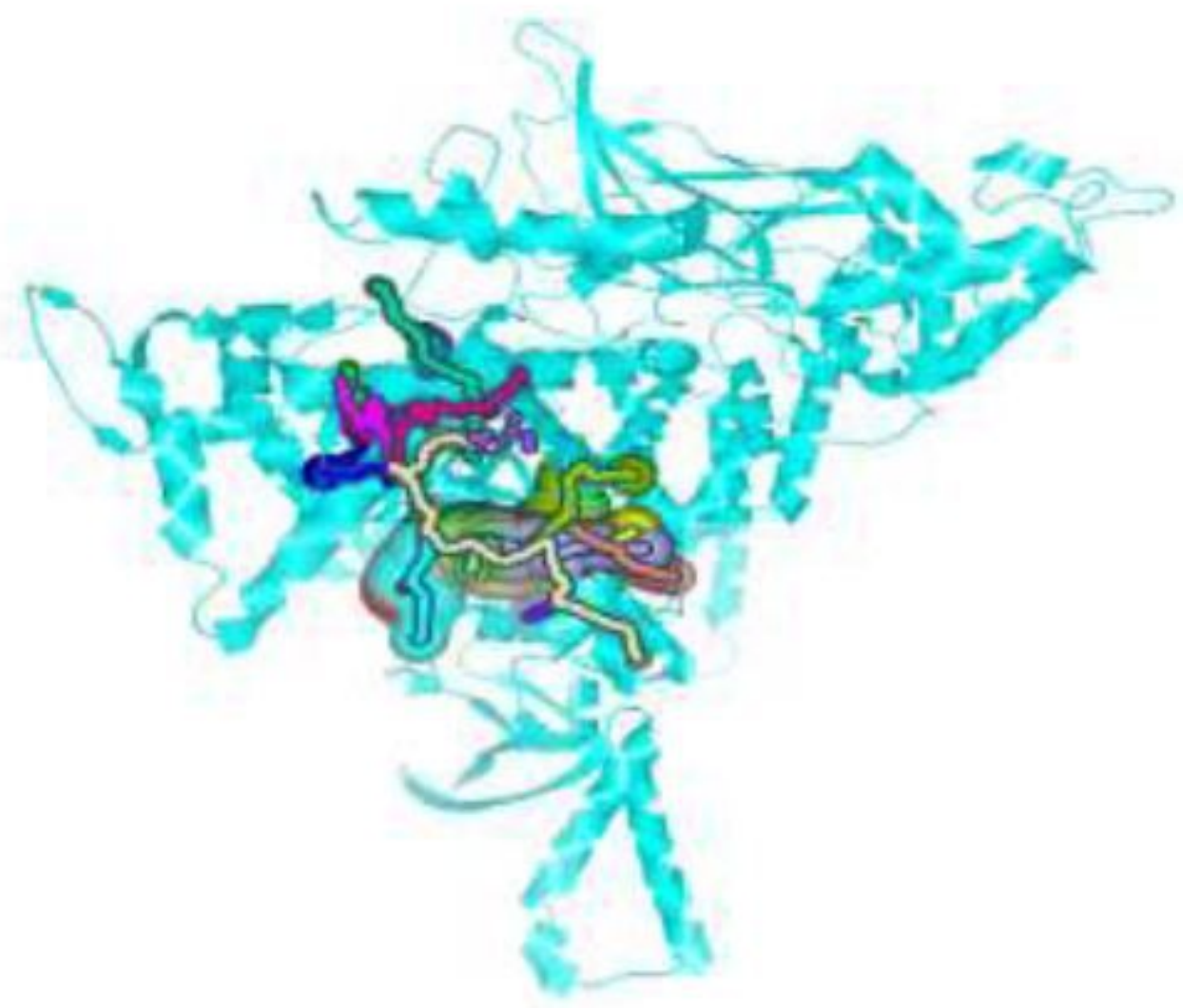

Figure 9

Three-dimensional simulation of ligand channels 